\title{
Remarks on Algebraic Decomposition of Automata
}

\author{
by \\ A. R. MEYER \\ and \\ C. THOMPSON \\ Carnegie-Mellon University
}

ABSTRACT

\begin{abstract}
A version of the Krohn-Rhodes decomposition theorem for finite automata is proved in which capabilities as well as semigroups are preserved. Another elementary proof of the usual Krohn-Rhodes theorem is also presented.
\end{abstract}

\section{Introduction}

The constructive half of Krohn and Rhodes' decomposition theorem for finite automata states that any finite automaton can be simulated by a cascade of reset and permutation automata. Moreover, the groups of the permutation automata in the cascade need be only simple groups which divide the semigroup of the original automaton. Assorted proofs of this theorem appear in $[1,2,3,4$, $5,7]$ and we include our own elementary proof in Section 5.

Our object in this paper is to supply the few extra steps necessary to prove a corrected version of a slightly stronger decomposition theorem stated by Hartmanis and Stearns [4]. This theorem appears in Section 3. In Section 4 we exhibit a counter-example to the theorem as originally stated by Hartmanis and Stearns, and briefly consider cascades of "half-reset" automata.

\section{Preliminaries}

Our notation follows Ginzburg [3]. In particular, function arguments appear on the left (so that $x f$ is the value of the function $f$ at the argument $x$ ). Composition of functions is designated by concatenation, with the leftmost function understood to apply first [so that $x f g=(x f) g$ ]. For a function $f$ and a set $S$, the restriction of $f$ to $S$ is denoted by $f \uparrow S$. The cardinality of $S$ is given by $|S|$. We use $\subset$ to mean improper inclusion. For a set $S$ and a family $\mathscr{F}$ of functions with domains including $S, S \mathscr{F}$ denotes $\{s f \mid s \in S, f \in \mathscr{F}\}$. As usual, $s \mathscr{F}$ means $\{s\} \mathscr{F}$, and $S f$ means $S\{f\}$.

A semi-automaton (or state machine) $A$ consists of a finite set $Q^{A}$ (of states), 
a finite set $\Sigma^{A}$ (of inputs), and a set of (transition) functions from $Q^{A}$ into $Q^{A}$ indexed by $\Sigma^{A}$. The function from $Q^{A}$ into $Q^{A}$ indexed by $\sigma \in \Sigma^{A}$ is $\sigma^{A}$. When the context is unambiguous, we shall frequently omit superscripts and identify $\sigma$ with $\sigma^{A}$.

Let $A$ and $B$ be semi-automata. $B$ is a subautomaton of $A$ if and only if $\Sigma^{B}$ $\subset \Sigma^{A}, Q^{B} \subset Q^{A}$ and $\sigma^{B}=\sigma^{A} \backslash Q^{B}$ for each $\sigma \in \Sigma^{B}$. A subautomaton $B$ of $A$ is non-trivial if $\Sigma^{B}=\Sigma^{A}$ and $\left|Q^{A}\right|>\left|Q^{B}\right|>1$. We say that $B$ is an image of $A$ if there are functions $\eta: Q^{A} \rightarrow Q^{B}$ and $\xi: \Sigma^{B} \rightarrow \Sigma^{A}$ such that $\eta$ is onto and $\eta \sigma^{B}=$ $(\sigma \xi)^{A} \eta$ for each $\sigma \in \Sigma^{B}$. The function $\eta$ is then called a homomorphism from $A$ (on)to $B$. We say that $A$ covers $B$, in symbols $A \geq B$, if and only if $B$ is an image of a subautomaton of $A$. Covering is transitive. We shall say that $A$ and $B$ are equivalent if and only if $A \geq B$ and $B \geq A$.

A partition $\pi$ of $Q^{A}$ is an admissible partition of $A$ if and only if for every $X \in \pi$ and $\sigma \in \Sigma^{A}$ there is a $Y \in \pi$ such that $X \sigma \subset Y$. The quotient semi-automaton $A / \pi$ (defined for admissible $\pi$ ) has state set $\pi$, inputs $\Sigma^{A}$, and transitions given by $X \sigma^{A / \pi}=Y$ where $Y$ is the (necessarily unique) element of $\pi$ such that $X \sigma \subset Y$. The semi-automaton $A / \pi$ is an image of $A$.

Given a (connecting) function $\omega: Q^{A} \times \Sigma^{A} \rightarrow \Sigma^{B}$, the cascade product $(A \circ B)_{\omega}$ is the semi-automaton with state set $Q^{A} \times Q^{B}$, inputs $\Sigma^{A}$, and transitions given by $(p, q) \sigma=(p \sigma, q((p, \sigma) \omega))$ for $(p, q) \in Q^{A} \times Q^{B}$ and $\sigma \in \Sigma^{A}$. We usually suppress mention of the connecting function and simply write $A \circ B$. Cascade product is associative in the sense that given $(A \circ B) \circ C$, there is an equivalent semiautomaton $A \circ(B \circ C)$. A cascade product of a sequence of three or more semiautomata is any parenthesization of the sequence into a cascade product of pairs of semi-automata.

If $B \geq D$, then for every connecting function $\omega$ there is a connecting function $\omega^{\prime}$ such that $(A \circ B)_{\omega^{\prime}} \geq(A \circ D)_{\omega}$. Similarly, if $A \geq C$, there is an $A^{\prime}$ equivalent to $A$ such that $A^{\prime} \circ B \geq C \circ B$.

We say that $A$ is a permutation semi-automaton if and only if every $\sigma \in \Sigma^{A}$ is a permutation of $Q^{A}$, and that $A$ is a reset if and only if every $\sigma \in \Sigma^{A}$ is a constant or identity function on $Q^{A}$; constant functions are also called resets. We call $A$ an identity semi-automaton if and only if every $\sigma \in \Sigma^{A}$ is the identity on $Q^{A}$.

We assume the reader is familiar with the elementary facts about grousp and semigroups. Let $S$ and $T$ be semigroups. Then $S$ is a subgroup of $T$ if and only if $S$ is a subsemigroup of $T$, and $S$ is (abstractly isomorphic to) a group. We say that $S$ divides $T$, in symbols $S \mid T$, if and only if $S$ is a homomorphic image of a subsemigroup of $T$. Division is transitive. By $T \rightarrow S$ we mean that $S$ is a homomorphic image of $T$, and by $S=T$ we mean that $S$ and $T$ are isomorphic. Most of the semigroups in this paper are transformation semigroups, but we use $\rightarrow$ and $=$ to mean homomorphism and isomorphism of abstract semigroups (though it will usually be clear when an abstract homomorphism is actually a transformation homomorphism). When $T$ is a group, $S \triangleleft T$ means $S$ is a normal subgroup.

The semigroup of a semi-automaton $A$ is the transformation semigroup generated by $\left\{\sigma^{A} \mid \sigma \in \Sigma^{A}\right\}$ under composition. The monoid $G^{A}$ is the semigroup of $A$ with $\Lambda^{A}$, the identity on $Q^{A}$, added if it is not already in the semigroup. If $A \geq B$, then $G^{B} \mid G^{A}$. The converse is not true. $A$ is an identity semi-automaton 
if and only if $\left|G^{A}\right|=1$, and a permutation semi-automaton if and only if $G^{A}$ is a group. Corresponding statements with the semigroup of $A$ in place of $G^{A}$ are not true.

\section{The Decomposition Theorem}

The following version of the Krohn-Rhodes decomposition theorem is proved in $[2,3,4,7]$.

THEOREM 1. For any semi-automaton $A$, there is a cascade product of semiautomata $A_{1}, A_{2}, \cdots, A_{n}$ which covers $A$ such that for all $i(1 \leq i \leq n)$ either

(1) $G^{A_{i}}$ is a simple group ${ }^{\dagger}$ and $G^{A_{i}} \mid G^{A}$, or

(2) $A_{i}$ is a two-state reset.

Moreover, if $G^{A}$ is a group, those $A_{i}$ which are resets will actually be identity semi-automata.

The components $A_{i}$ of the cascade covering $A$ are no more complicated than $A$, insofar as semigroups reflect the complexity of semi-automata. On the other hand, Theorem 1 does not prohibit the $A_{i}$ from being larger than $A$, and in fact the usual decomposition techniques applied to a five-state machine whose semigroup consists of resets and the alternating group of degree five yields an $A_{i}$ with sixty states. The following theorem eliminates this flaw.

Definition. Let $A$ be a semi-automaton. The completion of $A$ is the semiautomaton $\bar{A}$ such that $Q^{\bar{A}}=Q^{A}, \Sigma^{\bar{A}}=G^{A}$, and for $g \in G^{A}, g^{\bar{A}}=g$.

THEOREM 2. Theorem 1 is true when (l) is replaced by

(1') $G^{A_{i}}$ is a simple group and $\bar{A} \geq A_{i}$.

Clearly $G^{\vec{A}}=G^{A}$ and since $A \geq B$ implies $G^{B} \mid G^{A}$, we observe that Theorem 2 implies Theorem 1 .

We take Theorem 1 as our starting point and prove Theorem 2 from the following lemmas. A proof of Theorem 1 appears in Section 5.

LEMMA 1. Let $C$ be a semi-automaton such that $G^{C}$ is a group and $N \triangleleft G^{C}$. Let $\pi=\left\{q N \mid q \in Q^{C}\right\}$. Then $\pi$ is an admissible partition and $G^{C} / N \rightarrow G^{C / \pi}$.

Proof. The elements of $\pi$ are the orbits of $Q^{c}$ under the group of transformations $N$, and so $\pi$ is clearly a partition of $Q^{C}$. Moreover, $\pi$ is admissible: $N g=g N$ for all $g \in G^{C}$ since $N$ is normal, and so for all $q N \in \pi$ it follows that $(q N) g=q(N g)=q(g N)=(q g) N \in \pi$. Observe that the elements of $G^{C / \pi}$ are simply the elements of $G^{C}$ acting on $\pi$. Hence, $G^{C} \rightarrow G^{C / \pi}$ and $N$ is trivially included in the kernel of the homomorphism. Therefore $G^{C} / N \rightarrow G^{C / \pi}$.

LEMMA 2. Let $A$ be a semi-automaton such that $H$ is a simple group and and $H \mid G^{A}$. Then there is a semi-automaton $B$ such that $\bar{A} \geq B$ and $G^{B}=H$.

\footnotetext{
tWe remind the reader that $A_{i}$ is a permutation semi-automaton if and only if $G^{A_{i}}$ is a group.
} 
Proof. It is easy to show (cf. Ginzburg [3], Section 1.16) that if a group divides a semigroup, then the group is actually a homomorphic image of a subgroup of the semigroup. Let $K$ be a subgroup of $G^{A}$ of minimum size such that $K \rightarrow H$, and let $N \triangleleft K$ be the kernel of the homomorphism. Let $C$ be the subautomaton of $\bar{A}$ such that $Q^{C}=Q^{A} K$ and $\Sigma^{C}=K$. Then $G^{C}=K$ (as the reader may verify $)^{\dagger}$ and by Lemma $1, \pi=\left\{q N \mid q \in Q^{c}\right\}$ is an admissible partition of $C$. Finally, let $B=C / \pi$.

Clearly $\bar{A} \geq B$.

Lemma 1 also implies that $G^{C} / N \rightarrow G^{B}$. But $G^{C} / N=K / N=H$, and $H$ is simple, so that if $\left|G^{B}\right| \neq 1$, it must be that $G^{B}=H$ as required.

On the other hand, suppose that $\left|G^{B}\right|=1$. Then every element of $K=G^{C}$ acts as an identity on $\pi$, i.e., $(q N) k=q N$ for every $k \in K$ and $q N \in \pi$. For $q \in Q^{c}$, let $K_{q}=\{k \in K \mid q k=q\}$. Since $q \in q N=q N k$ for $q \in Q^{C}$, it follows that $K_{q}$ intersects every coset of $N$ in $K$, and so the restriction to $K_{q}$ of the canonical homomorphism from $K$ onto $K / N$ is also onto. Therefore, $K_{q} \rightarrow K / N=H$ (obviously $K_{q}$ is a group), and since $K$ is of minimum size, $K=K_{q}$ for all $q \in Q^{C}$. But this implies that $K=\left\{\Lambda^{c}\right\}$, which is absurd, since $H$ is a non-trivial image of $K$.

LEMMA 3. If $A$ and $B$ are semi-automata such that $G^{A}=G^{B}$, then there is a cascade product of copies of $\bar{B}$ and an identity semi-automaton which covers $A$.

Proof. For convenience assume that $Q^{B}=\{1,2, \cdots, n\}$. The cascade covering $A$ will consist of an identity machine with state set $Q^{A}$ and $n$ copies of $\bar{B}$, all acting in parallel. For $q_{0} \in Q^{A}, q_{i} \in Q^{B}, 1 \leq i \leq n$, and $g \in G^{B}$, the transitions in the cascade are defined by $\left(q_{0}, q_{1}, \cdots, q_{n}\right) g={ }_{\text {def }}\left(q_{0}, q_{1} g, \cdots, q_{n} g\right)$.

The states $q_{i} \in Q^{B}$ uniquely determine a function $f: Q^{B} \rightarrow Q^{B}$ by the condition $f(i)=q_{i}, 1 \leq i \leq n$. If $f \in G^{B}$, then the state $q_{0} f \in Q^{A}$ is also uniquely determined by the isomorphism between $G^{B}$ and $G^{A}$.

The states of the cascade which determine functions $f \in G^{B}$ obviously form a subautomaton of the cascade, and the mapping of $\left\langle q_{0}, q_{1}, \cdots, q_{n}\right\rangle$ to $q_{0} f$ defines a homomorphism from this subautomaton onto $\bar{A}$ (and hence onto $A$ ), as is easily verified.

Lemma 3 emphasizes the difficulty in interpreting the Krohn-Rhodes theorem as a "prime" decomposition theorem for machines (as opposed to semigroups). We might tentatively define $A$ to be prime if (1) $G^{A}$ is simple, and (2) $\bar{A} \geq B$ implies either $\bar{B} \geq A$ or $G^{B} \neq G^{A}$. Then there will be prime machines for the same simple group which are incomparable under covering. Lemma 3 then leads to the unsatisfactory situation of two primes each of which divides (is covered by) a power (cascade product of copies) of the other prime.

The proof of Theorem 2 is now straightforward. Each $A_{i}$ such that $G^{A_{i}}$ is a simple group can be covered according to Lemma 3, by a cascade of copies of $\bar{B}$ and an identity semi-automaton, for any $B$ such that $G^{B}=G^{A_{i}}$. Since $G^{A_{i}} \mid G^{A}$, Lemma 2 implies that such an automaton $B$ can be found for which $\bar{A} \geq B$ (and

${ }^{\dagger}$ This is not quite immediate, since one must argue that the identity of $K$ restricted to $Q^{C}$ is actually $\Lambda^{C}$. A proof appears in Ginzburg [3], Section 1.16. 
hence $\bar{A} \geq \bar{B}$ ). The identity semi-automata which are introduced can trivially be replaced by cascades of two-state identity semi-automata, and the proof is complete.

Hartmanis and Stearns' notion that " $A$ has the capability of $B$ " is equivalent to $\bar{A} \geq B$. Theorem 2 above is thus a restatement of Theorem 7.10 of Hartmanis and Stearns [4], except that their Theorem 7.10 contains the additional assertion that $\bar{A} \geq A_{i}$ even when $A_{i}$ is a reset. This is false, as we show in the next section.

\section{Half-resets}

Let $R_{0}$ be the semi-automaton whose state set and input set equals $\{0,1\}$ and whose transitions are given by ordinary multiplication. Any semi-automaton covered by $R_{0}$ will be called a half-reset. Except for permutation semi-automata, every semi-automaton has the capability of $R_{0}$.

Definition. Let $A$ be a semi-automaton and let $p, q \in Q^{A}$. Then $q$ is accessible from $p$ if and only if $q=p g$ for some $g \in G^{A}$, and $A$ is partially ordered (p.o.) if and only if accessibility is a partial order on $Q^{A}$.

$R_{0}$ is trivially p.o., and it is easy to show that if $A$ is p.o. and $A \geq B$, then $B$ is p.o. Likewise, if $A$ and $B$ are p.o., then so is $A \circ B$. Conversely, if $A$ is p.o. (and not already a half-reset), then $A$ has a non-trivial subautomaton which is a halfreset. We let the reader convince himself that $A$ can then be covered by a p.o. semi-automaton with one fewer state followed by a half-reset (cf. Method I of Section 5). In short, we have

THEOREM 4. A semi-automaton is covered by a cascade of half-resets if and only if it is partially ordered.

The regular events associated with p.o. semi-automata are obviously finite unions of events of the form $F_{1}^{*} \sigma_{1} F_{2}^{*} \sigma_{2} \cdots F_{n}^{*}$ such that $F_{i}$ is a finite set of input symbols and $\sigma_{i} \notin F_{i}(1 \leq i \leq n)$. These events form a Boolean algebra, and can also be characterized by an inductive definition resembling that of the starfree events [6]. One can also define partially ordered semigroups in the obvious way, and conclude that $A$ is p.o. if and only if $G^{A}$ is p.o.

Consider a semi-automaton $A$ with state set $\{1,2,3\}$ and inputs $x$ and $y$ such that $1 x=2,2 y=1$ and the remaining transitions lead to 3 . No non-trivial groups divide $G^{A}$, so that in the decomposition of $A$ satisfying Theorem 2 , only two-state resets appear. By Theorem 4 , not all of these two-state resets can be half-resets (because states 1 and 2 are mutually accessible, i.e., $A$ is not p.o.). But the only two-state resets covered by $\bar{A}$ are half-resets (as can be verified by exhaustion), and so $A$ cannot have the capability of all the components in its decomposition.

\section{Proof of Theorem 1}

There are at least three elementary proofs of Theorem 1 in the literature: Ginzburg's [3] corrected version of Zeiger's proof using set systems or covers, Arbib's [2] version of Krohn-Rhodes' proof, and the elegant proof of Zeiger 
[7]. Nevertheless, none of these proofs is very simple, ${ }^{\dagger}$ and so we feel another proof may still be of interest. Readers familiar with the other proofs will note that our Method I is essentially dual to that of Zeiger [7], and our Method III is almost the same as that of Arbib [2].

The following lemma appears in $[2,3,4]$ and we shall not repeat the proof.

LEMMA 4. Let $A$ be a permutation semi-automaton. Then $A$ can be covered by a cascade of two-state identity semi-automata and permutation semi-automata whose monoids are the factor groups in a composition series for $G^{A}$ (and hence are simple groups dividing $G^{A}$ ).

We refer to permutation semi-automata and two-state resets as basic. Theorem 1 follows immediately from Lemma 4 and

THEOREM 5. For any semi-automaton $A$, there is a cascade product of basic semi-automata $A_{1}, A_{2}, \cdots, A_{n}$ which covers $A$ such that for all $i(1 \leq i \leq n)$, if $G^{A_{i}}$ is a group, then $G^{A_{i}} \mid G^{A}$.

A natural way to prove Theorem 5 is to show that any semi-automaton can be covered by a product of two "smaller" semi-automata, and then use induction. (A disadvantage of the proof using set systems [3,4] is that it does not conform to this description.) The proper interpretation of "smaller" is necessarily a little devious.

Definition. For any transformation monoid $S, N(S)$ is the submonoid generated by the nonconstant $t^{\ddagger}$ (i.e., non-reset) elements of $S$. For any semiautomaton $A$, the measure of $A$ is the triple of positive integers $\mu(A)={ }_{\text {def }}$ $\left(\left|N\left(G^{A}\right)\right|,\left|Q^{A}\right|,\left|G^{A}\right|\right)$.

Measures will be well-ordered lexicographically in the usual manner:

Definition. If $x=\left(x_{1}, x_{2}, x_{3}\right)$ and $y=\left(y_{1}, y_{2}, y_{3}\right)$ are triples of integers, then $x>y$ if and only if $x_{1}>y_{1}$, or $x_{1}=y_{1}$ and $x_{2}>y_{2}$, or $x_{1}=y_{1}$ and $x_{2}=y_{2}$ and $x_{3}>y_{3}$.

LEMMA 5. For any semi-automaton $A$ which is not basic, there are semiautomata $B$ and $C$ such that

(1) $B \circ C \geq A$,

(2) $N\left(G^{B}\right) \mid G^{A}$, and either $\mu(B)<\mu(A)$ or $B$ is basic, and

(3) $N\left(G^{C}\right) \mid G^{A}$ and $\mu(C)<\mu(A)$.

$\dagger$ The proof of Zeiger [7] is given in only two and a half pages, and separates non-permutation semi-automata into only two cases. Unfortunately, Zeiger's remark that his method applies to permutation-reset semi-automata is false, as can be seen by applying it to any permutation-reset semi-automaton. Moreover, a semi-automaton with state set $\{1,2,3\}$, reset inputs to each state, and an additional input leaving states 1 and 3.fixed and sending state 2 to state 3 is a counter-example to Zeiger's assertion that his second method reduces the number of non-permutation, non-reset elements. This counter-example invalidates the proof that his method terminates. When these errors are corrected, Zeiger's proof turns out to be no simpler than ours.

${ }^{\ddagger}$ By convention $N(S)$ is the identity when $S$ acts on a singleton set. 
Proof of Theorem 5. Let $A$ be a semi-automaton. If $A$ is basic (and in particular if $\mu(A)=(1,1,1)$ is minimum), then Theorem 5 is trivially true. Proceeding by (transfinite) induction, suppose that Theorem 5 is true for all semi-automata with measures smaller than $\mu(A)$. Theorem 5 is then true by hypothesis for the semi-automata $B$ and $C$ produced by Lemma 5 . Let $B_{i}, 1 \leq i \leq n$, be the basic semi-automata in the cascade covering of $B$, and likewise for $C_{i}, 1 \leq i \leq m$. Since $B \circ C \geq A$, a cascade of the $B_{i}$ (or semi-automata equivalent to the $B_{i}$ ) followed by the $C_{i}$ covers $A$. Suppose $G^{B_{i}}$ is a group, then $G^{B_{i}} \mid G^{B}$. But if a group $G$ divides a transformation monoid $S$, then it must be that $G \mid N(S)$. Hence $G^{B_{i}}$. $N\left(G^{B}\right)$, by Lemma 5 we have $N\left(G^{B}\right) \mid G^{A}$, and by transitivity we have $G^{B_{i}}\left|G^{A}\right|$ The same reasoning applies to the $C_{i}$, and it follows that Theorem 5 is true for $A$.

Proof of Lemma 5. We describe three decomposition methods, one of which will yield appropriate $B$ and $C$ for any semi-automaton $A$ which is not basic.

Definition. For any semi-automaton $A$, let $N(A)$ be the subautomaton of $A$ obtained by eliminating all reset inputs from $\Sigma^{A}$.

Method I. $N(A)$ has a non-trivial subautomaton.

Let $Q^{C}$ equal the states of the non-trivial subautomaton of $N(A)$, and let $Q^{B}=\left(Q^{A}-Q^{C}\right) \cup\{d\}$ for $d \notin Q^{A}$. Transitions in $B \circ C$ are given by:

$$
(b, c) \sigma= \begin{cases}(b \sigma, c) & \text { if } b \neq d \text { and } b \sigma \notin Q^{c} \\ (d, b \sigma) & \text { if } b \neq d \text { and } b \sigma \in Q^{c} \\ (r, c) & \text { if } \sigma \text { is a reset to } r \in Q^{A}-Q^{c} \\ (b, c \sigma) & \text { otherwise. }\end{cases}
$$

Since $Q^{C}$ is the state set of a subautomaton of $N(A)$, it is closed under nonreset inputs. Hence the fourth case applies only when $b=d$ and $c \sigma \in Q^{C}$, so that the transitions of $B \circ C$ are well defined.

When $b \neq d$ map $(b, c)$ to $b$, and when $b=d$ map $(b, c)$ to $c$. This mapping defines a homomorphism from $B \circ C$ onto $A$ (as is immediately verified by checking the four types of transitions in $B \circ C$ ), so that part (1) of the lemma is satisfied.

Note that the singletons in $Q^{A}-Q^{C}$ together with $Q^{C}$ form an admissible partition $\pi$ of $A$, and that $A / \pi$ is isomorphic to $B$. We conclude that $G^{A} \rightarrow G^{B}$ and consequently that $N\left(G^{B}\right) \mid G^{A}$. Moreover, $\left|Q^{B}\right|=\left|Q^{A}-Q^{C}\right|+1<\left|Q^{A}\right|$ since the sub-automaton on $Q^{C}$ is non-trivial. This guarantees that part (2) is satisfied.

The only non-identity, non-reset transitions in $G^{C}$ arise from the fourth case in the definition of transitions of $B \circ C$. It follows that $N\left(G^{C}\right)=\left\{g \backslash Q^{C} \mid g \in N\left(G^{A}\right)\right\}$. Hence $N\left(G^{A}\right) \rightarrow N\left(G^{C}\right)$, and since $\left|Q^{C}\right|<\left|Q^{A}\right|$, part (3) is satisfied.

Method II. $G^{A}$ contains a non-identity permutation.

Let $P$ be the subgroup of $G^{A}$ generated by the permutations, and let $T$ be $G^{A}-P$. Note that $T \neq \varnothing$ (otherwise $G^{A}$ is a group and $A$ is basic) and that $T$ is a two-sided ideal. Let $Q^{B}=P$, and $Q^{C}=Q^{A}$. Transitions in $B \circ C$ are given by:

$$
(p, q) \sigma= \begin{cases}(p \sigma, q) & \text { if } \sigma \in P, \\ \left(p, q p \sigma p^{-1}\right) & \text { if } \sigma \in T .\end{cases}
$$


Since $G^{A}$ is the disjoint union of $P$ and $T$, the transitions of $B \circ C$ are well defined. The mapping of $(p, q)$ to $q p$ defines a homomorphism from $B \circ C$ onto $A$.

Clearly $G^{B}=P$, so that $N\left(G^{B}\right) \mid G^{A}$ and $B$ is basic. Likewise $G^{C}=T$, so that $N\left(G^{C}\right) \mid G^{A}$ and $N\left(G^{C}\right)$ does not contain the non-identity permutation in $N\left(G^{A}\right)$. Therefore $\mu(C)<\mu(A)$.

Method III. $G^{A}=V \cup T$ where $V$ is a subsemigroup such that $|N(V)|<$ $\left|N\left(G^{A}\right)\right|$ and $T$ is a proper left ideal of $G^{A}-\{\Lambda\}$.

Let $Q^{B}=V$ and $Q^{C}=Q^{A}$. Transitions in $B \circ C$ are given by:

$$
(v, q) \sigma= \begin{cases}(v \sigma, q) & \text { if } \sigma \in V-T, \\ (\Lambda, q v \sigma) & \text { if } \sigma \in T .\end{cases}
$$

The mapping of $(v, q)$ to $q v$ defines a homomorphism from $B \circ C$ onto $A$.

Clearly $G^{C}=T \cup\{\Lambda\}$, so that $G^{C} \mid G^{A}$ and $N\left(G^{C}\right) \leqq N\left(G^{A}\right)$. Moreover, $Q^{C}=$ $Q^{A}$ and $\left|G^{C}\right|<\left|G^{A}\right|$ because $T$ is proper. Hence $\mu(C)<\mu(A)$.

Note that $N\left(G^{B}\right)$ is a submonoid (generated by $\left.V-T\right)$ of $V$ acting on itself by right multiplication, and so $N\left(G^{B}\right) \mid V$. Moreover, any $r \in V$ which is a reset (on $\left.Q^{A}\right)$ is certainly a reset when $V$ acts on itself. Therefore $N\left(G^{B}\right)$ is isomorphic to a submonoid of $N(V)$, and we actually have $N\left(G^{\boldsymbol{B}}\right) \mid N(V)$. In particular, $\left|N\left(G^{\boldsymbol{B}}\right)\right|$ $\leq|N(V)|$. By hypothesis, $|N(V)|<\left|N\left(G^{A}\right)\right|$, so that $\mu(B)<\mu(A)$.

Let $A$ be a semi-automaton such that neither Method I nor Method II applies to $A$, and such that $A$ is not basic. We claim that Method III applies to $A$, which completes the proof of Lemma 5.

To verify the claim, let $S=G^{A}-\{\Lambda\}$. Now $S$ is a subsemigroup because $G^{A}$ contains no non-identity permutations. There is a non-reset element $s \in S$ (otherwise $A$ is a reset and Method I applies). If $G^{A} S \cup$ \{resets\} $=S$, then $N(A)$ has a non-trivial subautomaton on the states in the range of $s$, and Method I applies. Therefore $G^{A} S \cup\{$ resets $\} \neq S$, and in particular $S$ has proper left ideals (e.g., $G^{A} s$ ).

Let $T$ be a maximal left ideal of $S$, and let $V=G^{A} x \cup\{\Lambda\}$ for any $x \in S-T$. Then $(V-\{\Lambda\}) \cup T$ is a left ideal of $S$ properly containing $T$, which implies $(V-\{\Lambda\}) \cup T=S$ and $V \cup T=G^{A}$. If $x=s$, we have observed that $V \cup$ \{resets\} $\neq G^{A}$, and so $|N(V)|<\left|N\left(G^{A}\right)\right|$. Alternatively, $x$ is a reset, hence $G^{A} x$ contains only resets, and $|N(V)|=1<\left|N\left(G^{A}\right)\right|$.

There are usually many ways to decompose a semi-automaton into two semi-automata with smaller measures, and it is far from clear which choices ultimately yield the most satisfactory decomposition into basic semi-automata. It may even be desirable at times to cover a semi-automaton with semi-automata which have larger measures (but which presumably are "smaller" in some more general sense).

\section{REFERENCES}

[1] M. Arbib (ed.), Algebraic Theory of Machines, Languages, and Semigroups, Academic Press, New York, 1968.

[2] M. ARBIB, Theories of Abstract Automata, Prentice-Hall, Englewood Cliffs, N.J., 1968.

[3] A. Ginzburg, Algebraic Theory of Automata, Academic Press, New York, 1968. 
A. R. Meyer And C. Thompson

[4] J. Hartmanis and R. E. Stearns, Algebraic Structure Theory of Sequential Machines, Prentice-Hall, Englewood Cliffs, N.J., 1966.

[5] K. Krohn and J. Rhodes, Algebraic theory of machines, I, Trans. Amer. Math. Soc. 116 (1965), 450-464.

[6] A. R. MeYER, A note on star-free events, J. Assoc. Comput. Machinery 16 (1969), 220-225.

[7] P. ZeIGer, Yet another proof of the cascade decomposition theorem for finite automata, Math. Systems Theory 1 (1967), 225-288.

(Received 1 June 1968) 